\title{
Selection of an optimal promoter for gene transfer in normal $B$ cells
}

\author{
MAGDALENA WINIARSKA ${ }^{1}$, DOMINIKA NOWIS ${ }^{2,3}$, MALGORZATA FIRCZUK $^{1}$, \\ AGNIESZKA ZAGOZDZON $^{1}$, MAGDALENA GABRYSIAK $^{1}$, RADOSLAW SADOWSKI $^{1}$, \\ JOANNA BARANKIEWICZ ${ }^{1}$, MICHAL DWOJAK ${ }^{1}$ and JAKUB GOLAB ${ }^{1}$
}

\footnotetext{
${ }^{1}$ Department of Immunology, Center of Biostructure Research; ${ }^{2}$ Genomic Medicine, Medical University of Warsaw;

${ }^{3}$ Laboratory of Experimental Medicine, Centre of New Technologies, Warsaw University, 02-097 Warsaw, Poland
}

Received June 20, 2016; Accepted November 11, 2016

DOI: $10.3892 / \mathrm{mmr} .2017 .6974$

\begin{abstract}
Gene transfer into normal quiescent human B cells is a challenging procedure. The present study aimed to investigate whether it is possible to increase the levels of transgene expression by using various types of promoters to drive the expression of selected genes-of-interest. To produce lentiviral particles, the present study used the 2nd generation psPAX2 packaging vector and the vesicular stomatitis virus -expressing envelope vector pMD2.G. Subsequently, lentiviral vectors were generated containing various promoters, including cytomegalovirus (CMV), elongation factor-1 alpha (EF1 $\alpha)$ and spleen focus-forming virus (SFFV). The present study was unable to induce satisfactory transduction efficiency in quiescent normal B cells; however, infection of normal B cells with Epstein-Barr virus resulted in increased susceptibility to lentiviral transduction. In addition, the SFFV promoter resulted in a higher level of transgene expression compared with CMV or EF1a promoters. As a proof-of concept that this approach allows for stable gene expression in normal B cells, the present study used bicistronic lentiviral vectors with genes encoding fluorescent reporter proteins, as well as X-box binding protein-1 and binding immunoglobulin protein.
\end{abstract}

\section{Introduction}

Gene transfer into normal cells serves an important role in basic studies aiming to elucidate the fundamental processes taking place in living organisms, as well as in gene therapy and immunotherapy of human diseases. In the field of immunotherapy there remains a requirement to develop and optimize gene transfer into primary target cells. However, the majority

Correspondence to: Dr Magdalena Winiarska or Professor Jakub Golab, Department of Immunology, Center of Biostructure Research, Medical University of Warsaw, Banacha 1A, 02-097 Warsaw, Poland E-mail: magdalena.winiarska@wum.edu.pl

E-mail: jakub.golab@wum.edu.pl

Key words: B cells, transduction, gene transfer, lentivirus, promoter of basic studies are conducted with transformed cells, due to difficulties in achieving stable and efficient levels of gene expression in normal cells. Gene transfer into normal B cells is particularly difficult. Notably, B cells induced to express the antigen of interest can stimulate human antigen-specific cytotoxic T lymphocytes and are useful and convenient source of autologous antigen-presenting cells for examining human immune response. In addition, genetically modified B cells may be used to induce anti-tumor immune response, thus contribute to the development of novel strategies for treatment of cancer. Following gene transfer, B cells have additionally been successfully applied to induce tolerance and prevent autoimmune responses in animal models. Unfortunately, the use of nonviral methods for gene transfer into mature B cells is particularly ineffective, and retroviral transduction of quiescent mature B cells has not been reported in the literature. Therefore, lentiviruses have attracted significant interest, considering their ability to transduce nondividing cells $(1,2)$. Vectors based on murine leukemia virus (MLV) have previously been used to transfer genes into transformed B cells or B-cell precursors (3). However, only $1-4 \%$ of B cells were transduced with these vectors, and they were revealed to be ineffective in gene transduction of mature quiescent $B$ cells $(4,5)$. Even with lentiviruses, quiescent $\mathrm{B}$ cells require prior stimulation with cytokines, cell surface molecules or other factors to stimulate entry from $G_{0}$ to $G_{1}$ phase of the cell cycle, in order to become transduced. This is achieved by various methods, including B-cell activation via cluster of differentiation (CD)40, with either antibodies or feeder cells expressing CD40 ligand (CD40L) (6); cytokines, such as interleukin (IL)-4 and IL-21; CpG oligonucleotides (5); anti-CD20 antibodies (7); infection with Epstein-Barr virus (EBV); or a combination of these approaches (4). A major breakthrough was associated with the generation of pseudotyped lentiviral vectors. Lentiviral vectors pseudotyped with glycoprotein from the vesicular stomatitis virus (VSVG) or envelope proteins from g-retroviruses, such as MLV, have been reported to result in broad tropism in the majority of human cells (8); however, they were particularly ineffective in the case of B cells (6). Previous studies have revealed that VSVG-lentiviruses require low-density lipoprotein-receptor, which is inducible in numerous activated cells, but not in B cells $(9,10)$. Improved 
lentiviral pseudotypes have been designed by incorporating measle virus (MV) glycoproteins $\mathrm{F}$ and $\mathrm{H}$ into their surface (MV-lentiviruses) (11-13). These vectors target normal B cells through MV receptors, such as CD46 or signaling lymphocytic activation molecule $(14,15)$. Pseudotyped MV-lentiviruses have been reported to achieve $\sim 50 \%$ gene transfer efficiency in resting $\mathrm{B}$ cells (11); therefore, further improvements and optimization strategies are required. Efficient delivery of genes into primary human mature B cells may be used to investigate the functions of genes-of-interest (GOIs) in these cells. In addition, efficient gene delivery may result in the development of gene therapies, as well as the development of novel tools for B-cell immortalization, allowing for cloning of antigen-specific B-cell populations, which may be used in the production of monoclonal antibodies. Therefore, the aim of the present study was to further optimize the methods for efficient B-cell transduction by using various gene promoters to increase the levels of transgene expression.

\section{Materials and methods}

Cell culture. The Raji human Burkitt lymphoma cell line (American Type Culture Collection, Manassas, VA, USA) and human embryonic kidney (HEK)-293T cell line (Leibniz Institute DSMZ-German Collection of Microorganisms and Cell Cultures, Braunschweig, Germany) were cultured in RPMI 1640 medium (Sigma-Aldrich; Merck Millipore, Darmstadt, Germany). HT-1080 cells (American Type Culture Collection) were cultured in Dulbecco's modified Eagle's medium (DMEM; Sigma-Aldrich; Merck Millipore). The B95.8 human EBV-producing cell line was kindly provided by Professor U. Wojda (International Institute of Molecular and Cellular Biology, Warsaw, Poland); these cells were cultured in RPMI 1640 medium until they reached $\geq 2 \mathrm{mln} / \mathrm{ml}$ density. Subsequently, the supernatant was collected and filtered through a $0.45 \mu \mathrm{m}$ syringe filter (Promega Corporation, Madison, Wisconsin, USA), aliquoted and stored at $-80^{\circ} \mathrm{C}$ until further use. All media were supplemented with $10 \%$ heat-inactivated fetal bovine serum (FBS; HyClone; GE Healthcare Life Sciences, Logan, UT, USA), $100 \mu \mathrm{g} / \mathrm{ml}$ streptomycin, $100 \mathrm{U} / \mathrm{ml}$ penicillin and $250 \mathrm{ng} / \mathrm{ml}$ amphotericin B (Invitrogen; Thermo Fisher Scientific, Inc., Waltham, MA, USA). Cells were cultured at $37^{\circ} \mathrm{C}$ in a humidified atmosphere containing $5 \% \mathrm{CO}_{2}$, and were passaged approximately every other day.

$B$-cell isolation from blood and in vitro culture. Peripheral blood mononuclear cells (PBMCs) from healthy donors (recruited between September and November 2013, 3 males and 3 females aged 25-45 years old, blood collected via venipuncture from forearm) were isolated from full blood using Histopaque-1077 (Sigma-Aldrich; Merck Millipore) according to the manufacturer's protocol. B cells were isolated from PBMCs using the magnetic EasySep ${ }^{\mathrm{TM}}$ Human B Cell Enrichment Kit (STEMCELL Technologies Canada, Inc., Vancouver, BC, Canada) according to the manufacturer's protocol. Cells were cultured in Iscove's modified Dulbecco's medium (IMDM; Invitrogen; Thermo Fisher Scientific, Inc.) supplemented with $10 \%$ heat-inactivated FBS, $100 \mu \mathrm{g} / \mathrm{ml}$ streptomycin, $100 \mathrm{U} / \mathrm{ml}$ penicillin, and $250 \mathrm{ng} / \mathrm{ml}$ amphotericin $\mathrm{B}$ at $37^{\circ} \mathrm{C}$ in a humidified atmosphere containing $5 \% \mathrm{CO}_{2}$. The present study was approved by the Institutional Review Board of the Medical University of Warsaw (Warsaw, Poland) and was conducted according to the Declaration of Helsinki. Each patient provided written informed consent for the procedures. For co-culture experiments, HT-1080 pLVX-CD40L cells were incubated with mitomycin C $(10 \mu \mathrm{g} / \mathrm{ml}$; Sigma Aldrich; Merck Millipore) for $3 \mathrm{~h}$ at $37^{\circ} \mathrm{C}$, and were reseeded into 6 -well plates at a density of $4 \times 10^{5} /$ well. A total of $5 \times 10^{6} \mathrm{~B}$ cells at a density of $2.5 \times 10^{6} / 1 \mathrm{ml}$ were then placed onto the HT-1080 pLVX-CD40L cells in IMDM supplemented with $25 \mathrm{ng} / \mathrm{ml}$ IL-21 (PeproTech, Rocky Hill, NJ, USA).

Plasmids. A human CD40L coding sequence was amplified by polymerase chain reaction (PCR) from cDNA obtained from healthy donor PBMCs, and was cloned into a pLVX-internal ribosome entry site (IRES)-Puro vector (Takara Bio Europe, Saint-Germain-en-Laye, France) according to a standard protocol (16). Briefly, cells were washed with PBS, pelleted, and resuspended in $1 \mathrm{ml} \mathrm{TRIzol}{ }^{\circledR}$ reagent (Invitrogen; Thermo Fisher Scientific, Inc.) to extract total RNA, according to the manufacturer's protocol. First strand cDNA synthesis was then performed: $0.5 \mu \mathrm{g}$ total RNA was primed with oligo(dT) using AMV reverse transcriptase (EURx Ltd., Gdansk, Poland). PCR was performed using Mastercycler personal (Eppendorf Instrumente $\mathrm{GmbH}$, Hamburg, Germany) and Color Opti Taq polymerase (EURx Ltd.) using the following PCR cycling conditions: Initial denaturation at $94^{\circ} \mathrm{C}$ for $2 \mathrm{~min}$, followed by 30 cycles of denaturation at $94^{\circ} \mathrm{C}$ for $30 \mathrm{sec}$, annealing at $58^{\circ} \mathrm{C}$ for $30 \mathrm{sec}$, elongation at $72^{\circ} \mathrm{C}$ for $60 \mathrm{sec}$, and a final elongation step at $72^{\circ} \mathrm{C}$ for $10 \mathrm{~min}$. Amplification products were analyzed by $1 \%$ agarose gel electrophoresis. The following primers containing restriction sites for XhoI and BamHI on the forward and reverse primer, respectively were used: Forward, 5'-CCG ACTCGAGACCATGATCGAAACATACAACCAAACTTC TCC-3' and reverse, 5'-GCGGGGATCCTCAGAGTTTGA GTAAGCCAAAGGACG-3'. The sequence of the construct (pLVX-CD40L-IRES-Puro) was confirmed by Sanger sequencing using BigDyeTM Terminator Version 3.1 Ready Reaction Cycle Sequencing kit (Thermo Fisher Scientific, Inc.), according to the manufacturer's protocol. The human immunodeficiency virus (HIV)-spleen focus-forming virus (SFFV) -monomeric red fluorescent protein (mRFP)-woodchuck hepatitis virus posttranscriptional regulatory element (WPRE) plasmid was a generous gift by Professor Els Verhoeyen (Ecole Normale Supérieure de Lyon, Lyon, France) (12). The sequences encoding MCS-IRES-RFP, BiP-IRES-RFP, MCS-IRES-GFP and XBP1s-IRES-GFP were synthesized by Epoch Life Science, Inc. (Missouri City, TX, USA) and provided as inserts in pBluescript SK vectors. The inserts were subsequently cloned by ligation into the BamHI and XhoI sites of the HIV-SFFV-mRFP-WPRE plasmid. Sequences of the constructs were confirmed by Sanger sequencing.

Lentiviral transduction of target cells. For lentiviral production, HEK-293T cells were seeded into 6-well plates (4x $10^{5} /$ well) and were co-transfected with $2 \mu \mathrm{g}$ gene-of-interest (GOI)-containing vectors and components of 2 nd generation packaging vectors: $1.5 \mu \mathrm{g}$ psPAX2 packaging vector and $1 \mu \mathrm{g}$ pMD2.G envelope vector (both vectors were obtained 
Table I. Antibodies used for cell sorting.

\begin{tabular}{|c|c|c|c|c|c|}
\hline Antibody & Fluorochrome & Clone & Cat. no. & Dilution & Company \\
\hline CD19 & FITC & 4G7 & 345776 & $1: 10$ & BD Biosciences \\
\hline CD20 & FITC & L27 & 345792 & $1: 10$ & BD Biosciences \\
\hline $\mathrm{CD} 27$ & PE & L128 & 340425 & $1: 10$ & BD Biosciences \\
\hline CD138 & FITC & MI15 & 552723 & $1: 10$ & BD Biosciences \\
\hline CD20 & $\mathrm{PE}$ & $2 \mathrm{H} 7$ & 555623 & $1: 10$ & BD Biosciences \\
\hline $\operatorname{IgD}$ & FITC & Polyclonal & F0189 & $1: 10$ & Dako \\
\hline $\operatorname{IgM}$ & FITC & Polyclonal & F0058 & $1: 10$ & Dako \\
\hline $\operatorname{IgG}$ & FITC & Polyclonal & F0185 & $1: 10$ & Dako \\
\hline $\operatorname{IgA}$ & FITC & Polyclonal & F0188 & $1: 10$ & Dako \\
\hline CD40L & $\mathrm{PE}$ & $89-76$ & 555700 & $1: 10$ & BD Biosciences \\
\hline
\end{tabular}

CD, cluster of differentiation; Ig, immunoglobulin; FITC, fluorescein isothiocyanate; PE, phycoerythrin. BD Biosciences, La Jolla, CA, USA; Dako; Agilent Technologies, Santa Clara, CA, USA.

from Professor Didier Trono; École Polytechnique Fédérale de Lausanne, Lausanne, Switzerland), using GeneJuice ${ }^{\circledR}$ transfection reagent (EMD Millipore, Billerica, MA, USA), according to the manufacturer's protocol. The following lentiviral vectors were used in this study: pLVX-IRES-Puro and pLVX-IRES-ZsGreen1 (both from Clontech, Takara Bio Europe), pGIPZ (Open Biosystems; GE Healthcare Life Sciences), pLVTHM (AddGene, Inc., Cambridge, MA, USA). $72 \mathrm{~h}$ post-transfection, the lentivirus-containing medium was collected, filtered and added to the culture of target HT-1080 or human B cells with a multiplicity of infection (MOI) of 2 . MOI was determined using the Lenti-X p24 Rapid Titer kit (Clontech, Takara Bio Europe), according to the manufacturer's protocol. To select GOI-containing cells, either puromycin selection or sorting for fluorescent-positive cells using a FACSAria III cell sorter (BD Biosciences, La Jolla, CA, USA) was performed 7 days post-transduction. HT-1080 cells modified with pLVX-CD40L-IRES-Puro construct (HT-1080 pLVX-CD40L) were cultured in DMEM supplemented with $1 \mu \mathrm{g} / \mathrm{ml}$ puromycin (Sigma Aldrich; Merck Millipore).

Staining of surface antigens. Cells were incubated with saturating amounts of fluorochrome-conjugated antibodies (Table I) for $30 \mathrm{~min}$ at room temperature. Prior to analysis cells were washed and resuspended in PBS. Cells were analyzed using a FACScan (BD Biosciences).

EBV infection and generation of lymphoblastoid cell lines (LCL). The supernatant from B95.8 cells was collected and stored as aforementioned, and was thawed on the day of infection. Human B cells freshly isolated from buffy coats, as aforementioned, were seeded at a density of $5 \mathrm{mln} / \mathrm{ml}$ in IMDM in a T25 flask. Subsequently, $2 \mathrm{ml}$ fresh RPMI 1460 medium was added, followed by $2 \mathrm{ml} \mathrm{EBV-containing} \mathrm{B95.8}$ supernatant. $24 \mathrm{~h}$ post-infection the cells cultured at $37^{\circ} \mathrm{C}$ began to form clusters.

Fluorescent microscopy. Cell cultures were analyzed for the expression of fluorescent proteins (GFP and RFP) using an inverted fluorescence microscope (Eclipse TE-2000; Nikon
Corporation, Tokyo, Japan) equipped with Plan Fluor 10x/0.30 Ph1 DLL, S Plan Fluor ELWD 20x/0.45 Ph1 ADM and Plan Fluor ELWD 40x/0.60 Ph2 ADL objectives, together with UV-2E/C, B-2E/C, G-2E/C and Y-2E/C fluorescence filters. Images were captured using a QICAM FAST1394 12-bit digital CCD camera (Q Imaging, Surrey, BC, Canada) and were analyzed using Image-Pro Plus 7.0 software (Media Cybernetics, Inc., Rockville, MD, USA).

ELISA. The concentration of immunoglobulin (Ig)G in cell culture supernatants was evaluated using Human IgG ELISA kit (Koma Biotech, Seoul, Korea), according to the manufacturer's protocol. This assay allows for detection of $\mathrm{IgG}$ in a sample at the range of $1.95-125 \mathrm{ng} / \mathrm{ml}$.

Western blotting. Cells were washed with PBS, pelleted, lysed in a custom-made lysis buffer (50 mM HEPES pH 7.4, 1.0\% Triton X-100, $150 \mathrm{mM} \mathrm{NaCl}, 10 \%$ glycerol, $5 \mathrm{mM}$ EDTA) and separated by $10 \%$ SDS-PAGE. Protein concentration prior to electrophoresis was measured using the Bradford Protein Assay kit (Bio-Rad Laboratories, Inc., Hercules, CA, USA). Total proteins (10-20 $\mu \mathrm{g})$ were loaded onto each well. Separated proteins were transferred onto Protran nitrocellulose membranes (Schleicher \& Schuell BioScience GmbH, Dassel, USA). Membranes were blocked for $1 \mathrm{~h}$ at room temperature with 5\% non-fat milk in TBS-0.1\% Tween (TBST; see below). Membranes were then incubated with anti-XBP-1s (cat. no. 647502; 1:1,000; BioLegend, San Diego, CA, USA) overnight at $4{ }^{\circ} \mathrm{C}$ in the presence of $5 \%$ bovine serum albumin (Sigma Aldrich; Merck Millipore). After washing with TBST, membranes were incubated with anti-rabbit horseradish peroxidase (HRP)-conjugated secondary antibody (cat.no. 111-035-144; Jackson ImmunoResearch Laboratories, Inc., West Grove, PA, USA) for $1 \mathrm{~h}$ at room temperature. The chemiluminescence reaction for HRP was developed using a luminol-based chemiluminescence reagent SuperSignal West Pico (Thermo Fisher Scientific, Inc.) and blots were visualized using STELLA 8300 bioimager (Raytest, Straubenhardt, Germany). The blots were then re-probed with anti- $\beta$-actin peroxidase-purified immunoglobulin (clone AC-15; 1:50,000; 

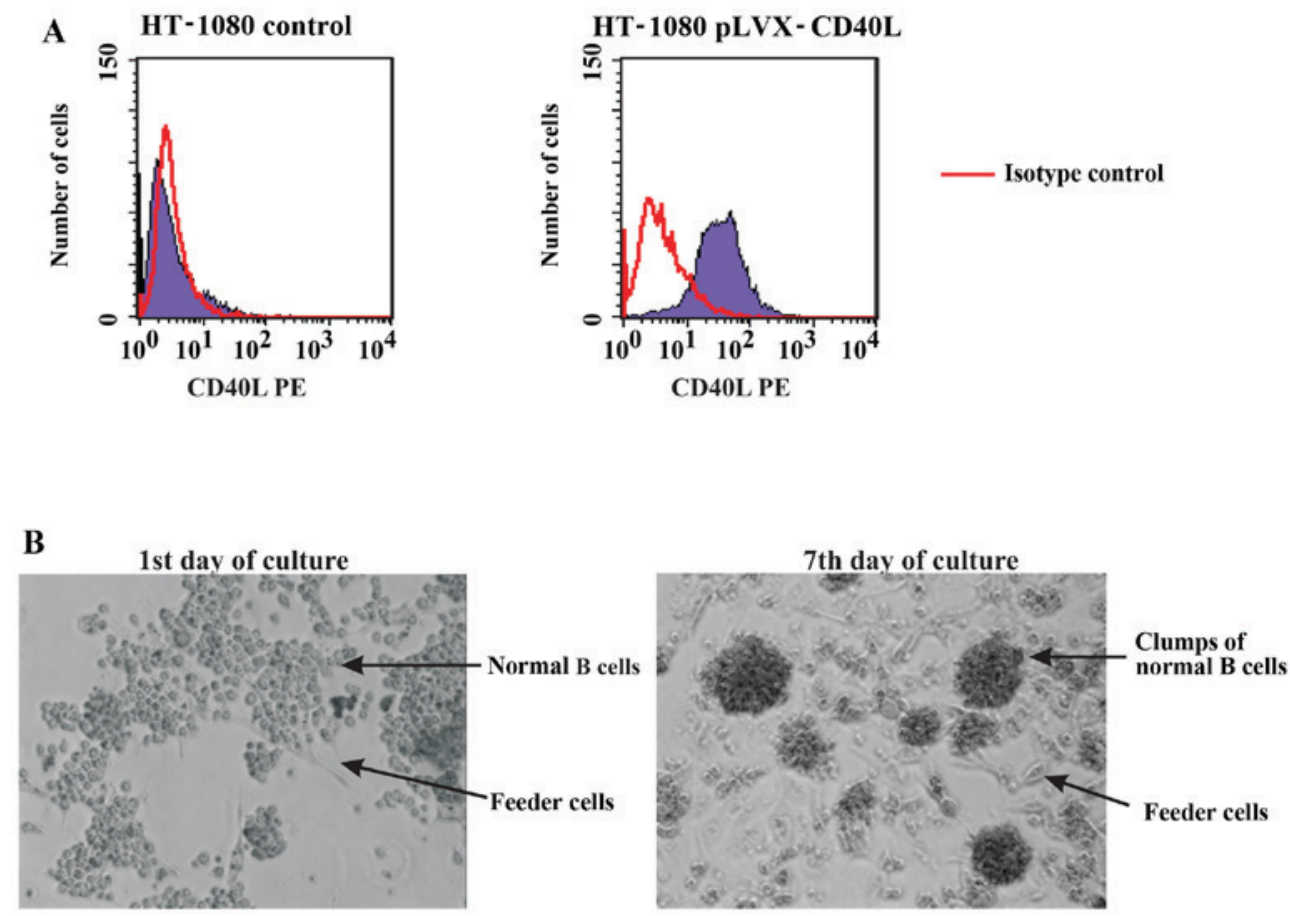

Figure 1. Culture of normal B cells with CD40L-expressing feeder cells. HT-1080 cells were modified to express CD40L, as confirmed by (A) flow cytometry. (B) HT-1080 pLVX-CD40L cells were used as feeder cells to stimulate the proliferation of normal B cells. Images were captured using an Nikon Eclipse TE2000-E microscope, magnification, x200. CD40, cluster of differentiation 40 ligand; PE, phycoerythrin.

Sigma Aldrich; Merck Millipore) for $45 \mathrm{~min}$ at room temperature.

\section{Results}

Normal B cells co-cultured with CD40L-expressingfeeder cells start to proliferate after 7 days of culture. The present study aimed to select optimal constructs and promoters for genetic modification of B cells. As a terminal cell type, B cells can usually only be cultured for a relatively short time. Therefore, to prolong the culture period B cells were co-cultured with feeder cells expressing human CD40L. Initially, HT-1080 cells were modified with an expression vector encoding CD40L (Fig. 1A). The CD40L coding sequence was amplified by PCR from the cDNA of healthy donor PBMCs, after which it was cloned into the pLVX-IRES-Puro vector and subsequently used to modify HT-1080 cells. Feeder cells were pretreated with mitomycin $\mathrm{C}$ to prevent their proliferation before starting the co-cultures. B cells co-cultured with feeder cells formed clumps of proliferating cells that were viable for $\geq 14$ consecutive days (Fig. 1B).

$B$ cells activated with $C D 4 O L$ and $I L-21$ are resistant to transduction. To determine the levels of transgene expression in B cells three bicistronic plasmids were used that encode various GFPs under the control of different promoters, namely pGIPZ [cytomegalovirus (CMV) promoter; turbo GFP, which is an improved variant of the green fluorescent protein CopGFP], pLVTHM [elongation factor 1 alpha (EF1 $\alpha)$ promoter; GFP) and pLVX-IRES-ZsGreen1 (CMV promoter; ZsGreen1, which is a human codon-optimized variant of ZsGreen) (Fig. 2A). Independent of the vectors used in the present study, a very low level of transgene expression was detected in B cells; expression did not exceed 10\%, as assessed with flow cytometry 7 days post-transduction (Fig. 2B). Notably, concomitant transduction of Raji lymphoma cells with pLVX ZsGreen1, pGIPZ and pLVTHM proved to be effective; the levels of transgene expression ranged between 14.22 and $40.25 \%$ (Fig. 2C).

EBV infection of B cells generates LCLs. Since Raji cells carry the latent EBV genome and are positive for EBV nuclear antigen 1, and EBV infection has previously been described to increase the sensitivity of $\mathrm{B}$ cells to genetic modification (17), the present study infected normal B cells with EBV to determine whether this would allow for increased levels of transgene expression with lentiviral vectors. Freshly isolated human B cells were mixed with supernatants collected from B95.8 human EBV-producing cells, in order to allow B cell infection and immortalization. Transformation of B cells with EBV resulted in the generation of LCLs. These cells were highly variable in shape and grew in clumps. The cells expressed antigens characteristic for normal B lymphocytes, such as CD19 and CD20 (Fig. 3A). Furthermore, EBV-infected $\mathrm{B}$ cells displayed memory phenotype, as they were positive for $\mathrm{CD} 27$. The expression of $\mathrm{CD} 138$, which is an antigen that is present during activation and differentiation of $\mathrm{B}$ cells and is specific for terminally differentiated B cells, was variable in tested LCLs (Fig. 3A). In addition, the membrane expression of Igs was determined, and the results indicated that LCLs do not express membrane IgD. However, $~ 60 \%$ of LCLs expressed membrane $\mathrm{IgG}$, whereas $\sim 40 \%$ expressed membrane IgA and membrane IgM (Fig. 3B). These results confirmed the findings of previous studies $(18,19)$, and suggested that EBV infection 
A

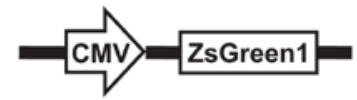

B

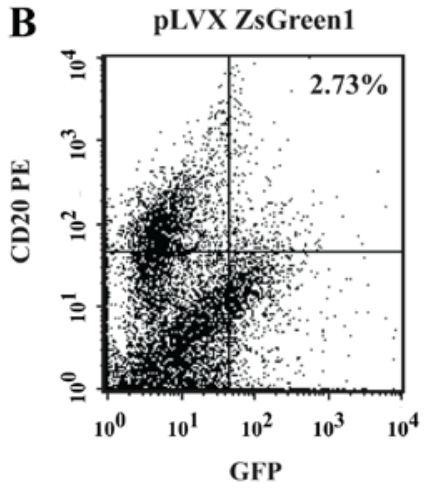

C

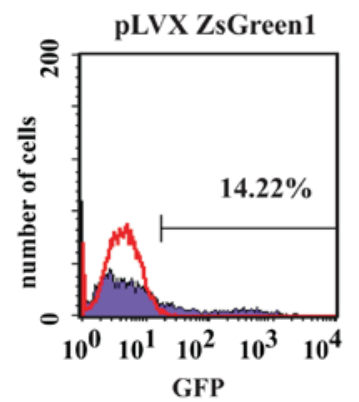

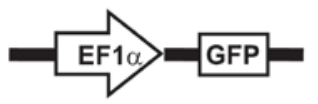

pLVTHM
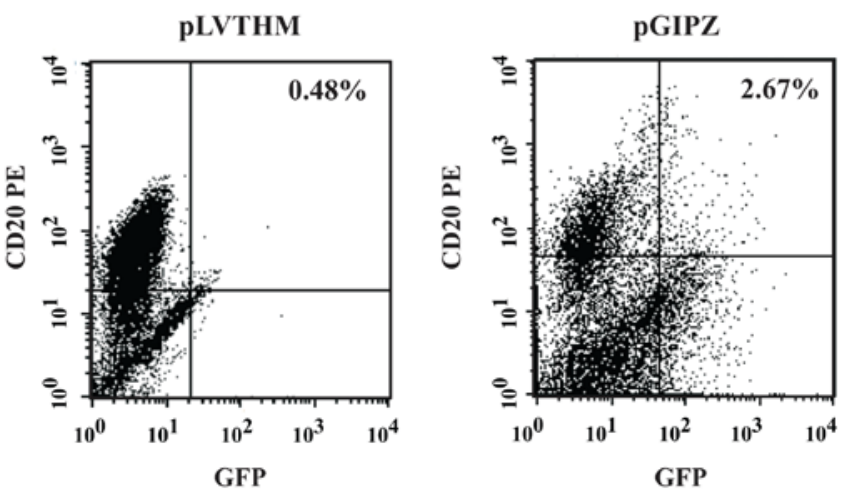

non-transduced cells

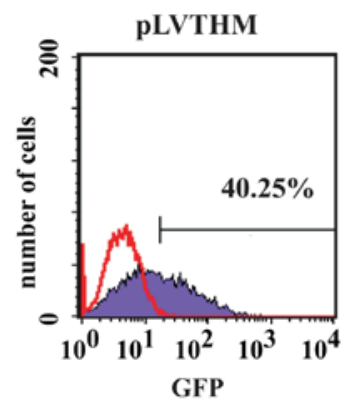

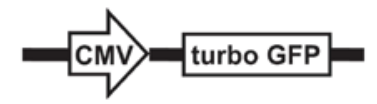

GFP

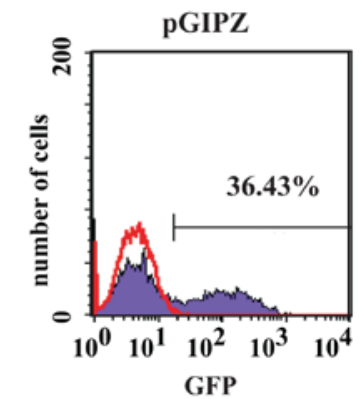

Figure 2. Lentiviral modification of normal B cells and Raji cells. (A) Three vectors (pLVX ZsGreen1, pLVTHM and pGIPZ) were used for lentiviral modification of (B) normal B cells or (C) Raji cells. The percentage of GFP-positive cells was determined using flow cytometry. Co-cultures of normal B cells and feeder cells were additionally stained with anti-CD20 antibody to distinguish B cells from feeder cells. GFP, green fluorescent protein; CMV, cytomegalovirus; EF1 $\alpha$, elongation factor 1 alpha; CD20, cluster of differentiation 20; PE, phycoerythrin.

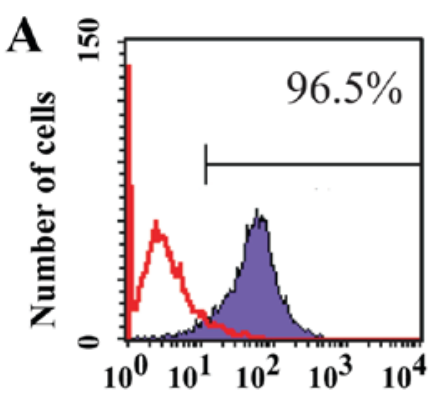

CD19 FITC

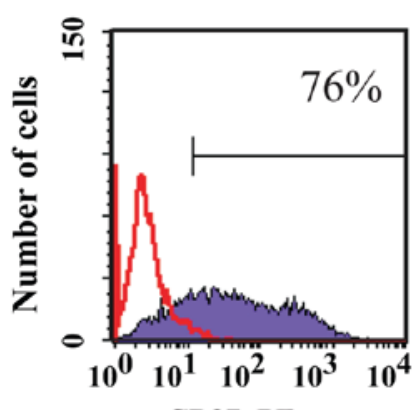

CD27 PE

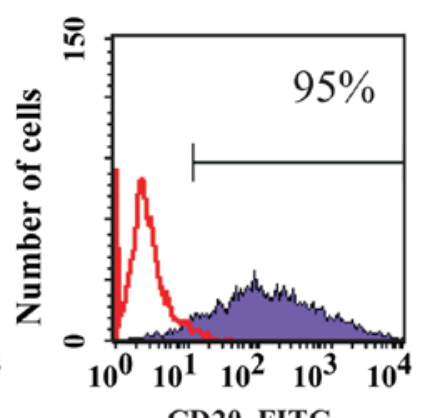

CD20 FITC

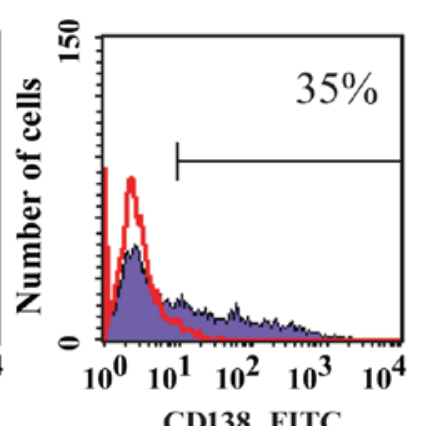

CD138 FITC

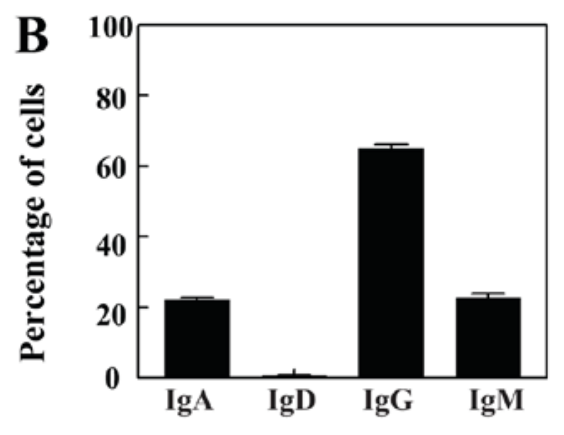

Figure 3. Characteristics of Epstein-Barr virus-infected B cells. Surface expression of (A) CD19, CD20, CD27 and CD138, and (B) Igs (IgA, IgD, IgG and IgM) in lymphoblastoid cell lines was determined by flow cytometry with fluorochrome-conjugated antibodies. CD, cluster of differentiation; Ig, immunoglobulin; FITC, fluorescein isothiocyanate; PE, phycoerythrin. 
non-transduced cells
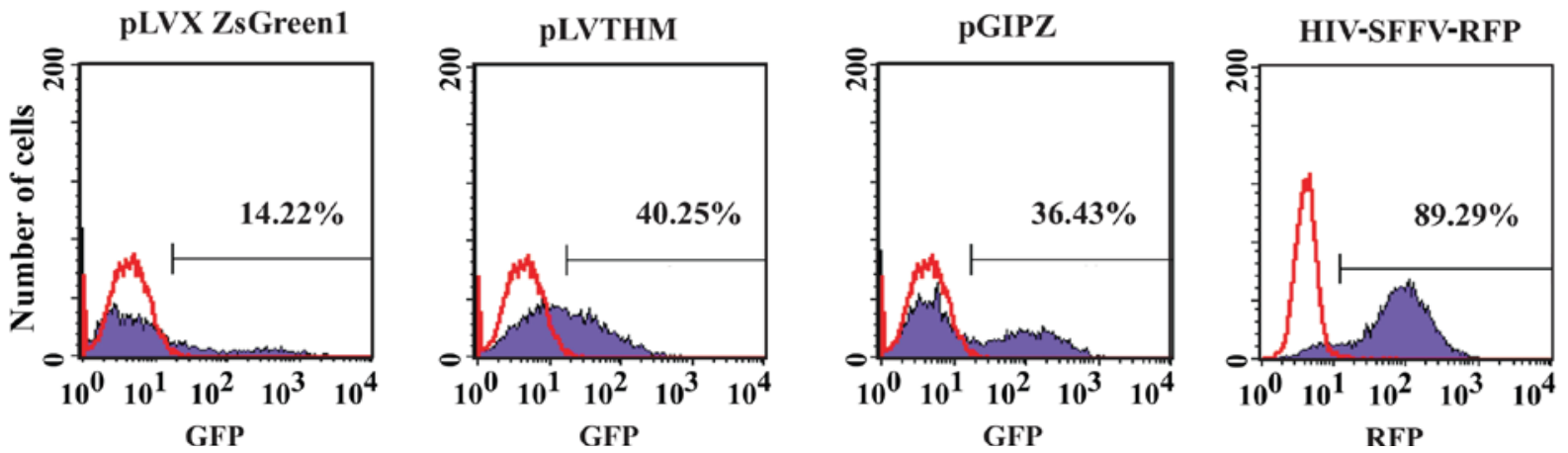

Figure 4. Transduction of Raji cells with plasmids containing various promoters. Raji lymphoma cells were modified with pLVX ZsGreen1, pLVTHM, pGIPZ and HIV-SFFV-RFP. The percentage of fluorescent (GFP- or RFP-positive) cells was determined using flow cytometry. GFP, green fluorescent protein; RFP, red fluorescent protein; HIV, human immunodeficiency virus; SFFV, spleen focus-forming virus.
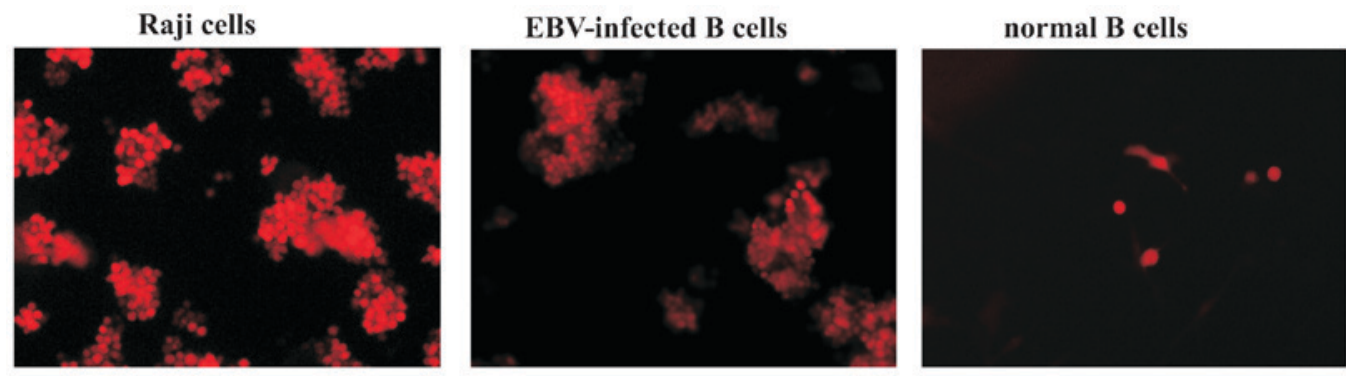

RFP
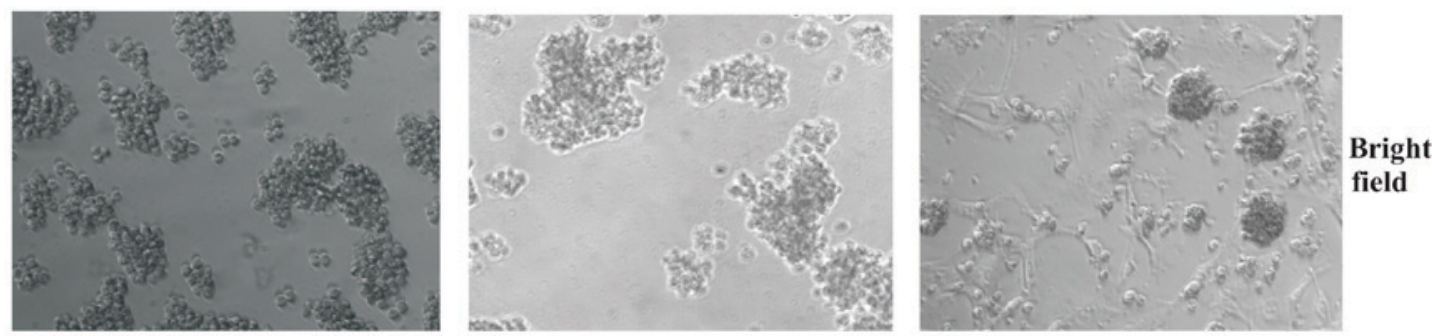

Figure 5. Transduction of Raji cells, LCLs and normal B cells with HIV-SFFV-mRFP-WPRE vector. Raji cells, LCLs and normal B cells were modified with a HIV-SFFV-mRFP-WPRE vector. After 7 days, cells were analyzed for RFP expression using fluorescent microscopy (Nikon Eclipse TE2000-E microscope; magnification, x200). mRFP, monomeric red fluorescent protein; LCLs, lymphoblastoid cell lines; HIV, human immunodeficiency virus; SFFV, spleen focus-forming virus; WPRE, woodchuck hepatitis virus posttranscriptional regulatory element; EBV, Epstein-Barr virus.
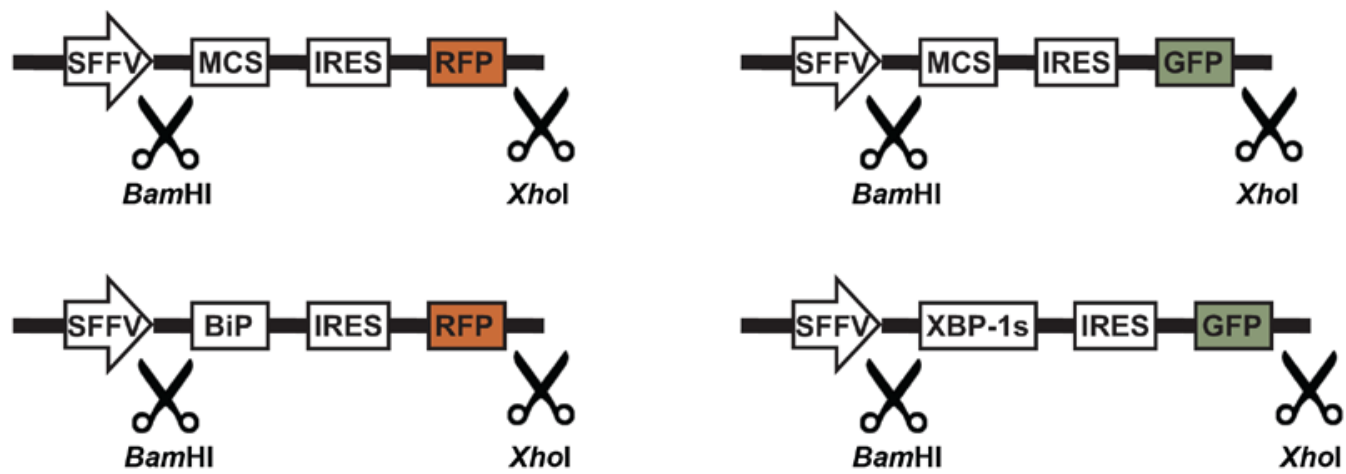

Figure 6. Constructs created and used for sorting transduced cells. HIV-SFFV-mRFP-WPRE vector was used to create bicistronic lentiviral vectors with genes encoding fluorescent reporter proteins (GFP or RFP), as well as XBP-1 and BiP. GFP, green fluorescent protein; RFP, red fluorescent protein; IRES, internal ribosome entry site; SFFV, spleen focus-forming virus; MCS, multiple cloning site; XBP-1, X-box binding protein 1; BiP, binding immunoglobulin protein.

expands the $\mathrm{CD} 27^{+}$memory cell pool, and the majority of these cells exhibited a switch in isotype to IgG or IgA. In addition,
LCLs were tested for the secretion of Igs; the mean output was $0.004 \mathrm{ng}$ IgG per cell per day (data not shown). 
non-transduced cells
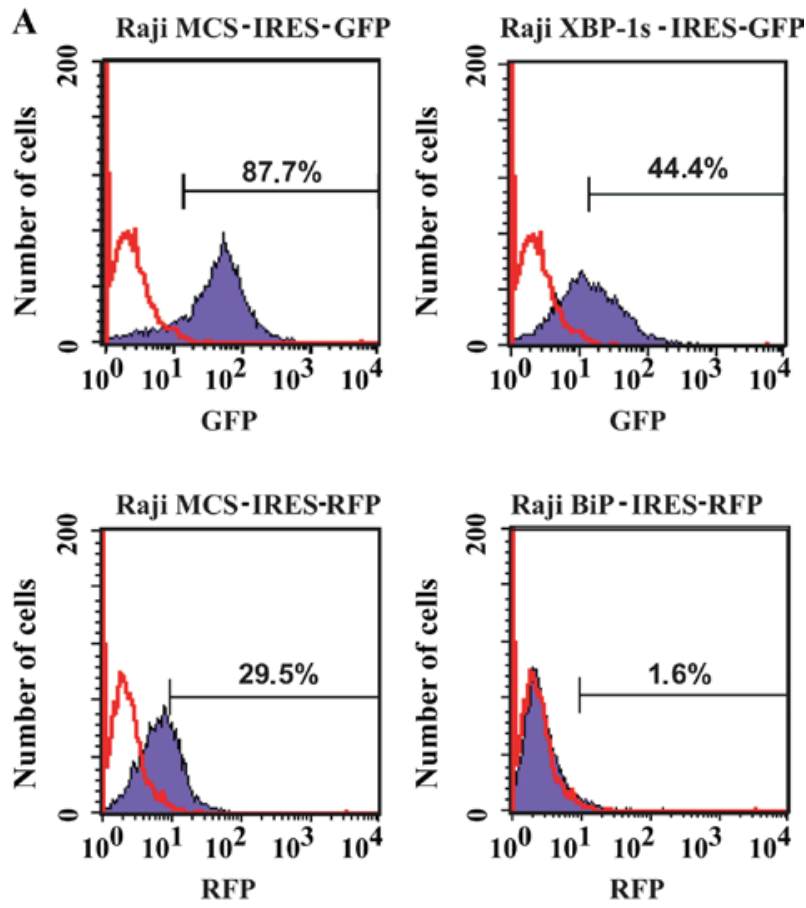

B
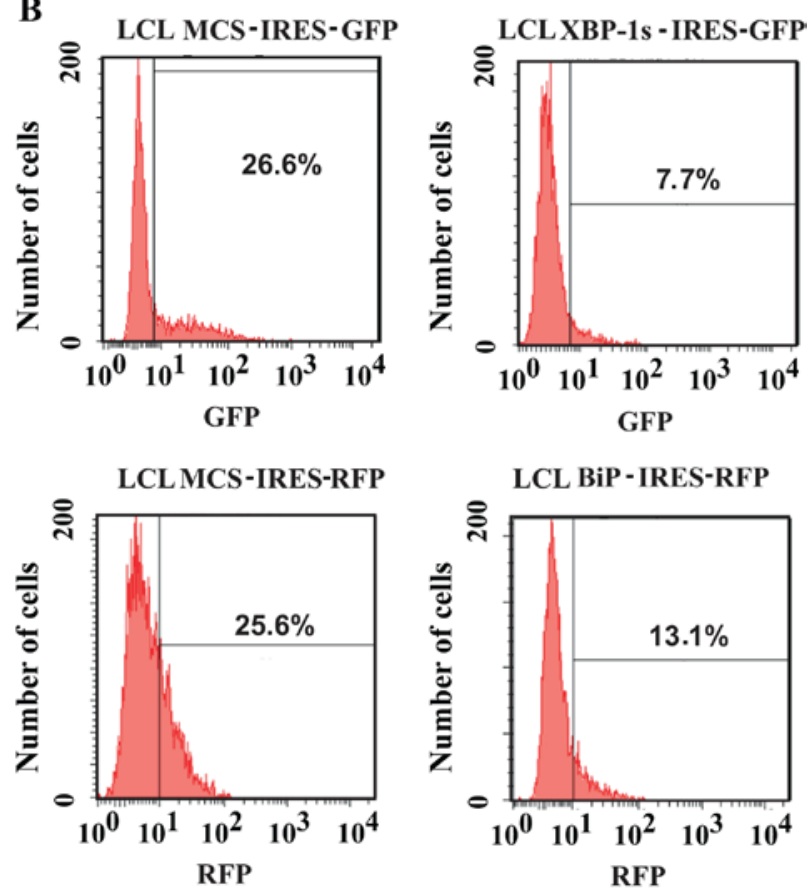

Figure 7. Transduction of Raji or Epstein-Barr virus-infected B cells with created constructs. (A) Raji cells or (B) LCLs were transduced with selected vectors, and after 10 days were analyzed with flow cytometry for the expression of fluorescent markers. Cells were sorted using a flow cytometer. LCLs, lymphoblastoid cell lines; RFP, red fluorescent protein; GFP, green fluorescent protein; IRES, internal ribosome entry site; MCS, multiple cloning site; $\mathrm{XBP}-1, \mathrm{X}$-box binding protein 1; BiP, binding immunoglobulin protein.

SFFV promoter provides the best level of transgene expression in Raji cells and LCLs. Since CMV and EF1 $\alpha$ promoters allowed for $\leq 40.25 \%$ transgene expression in Raji cells further experiments were conducted using the SFFV promoter. The results demonstrated that $\mathrm{SFFV}$ was much stronger in driving

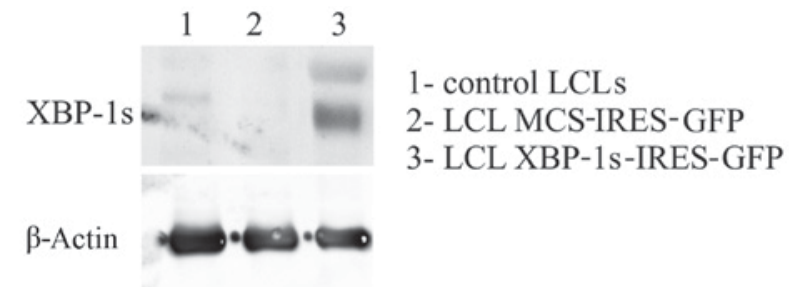

Figure 8. Sorting of Epstein-Barr virus-infected B cells following modification with XBP-1. LCLs modified to overexpress XBP-1s were sorted and subsequently analyzed using western blotting. $\beta$-actin served as a loading control. LCL, lymphoblastoid cell lines; IRES, internal ribosome entry site; GFP, green fluorescent protein; MCS, multiple cloning site; XBP-1, X-box binding protein 1.

transgene expression in human hematopoietic cells compared with other promoters, including CMV or EF1 $\alpha$ (12). Notably, the level of transgene expression in Raji cells was $>89 \%$ when the SFFV promoter was used (Fig. 4). Subsequently, the present study analyzed the levels of transgene expression in Raji cells, LCLs and normal CD40L-stimulated B cells using the HIV-SFFV-mRFP-WPRE vector. The SFFV promoter resulted in high levels of transgene expression in Raji and LCL cells, as determined by fluorescent microscopy; however, normal CD40L-stimulated B cells were still resistant to modification (Fig. 5).

Constructs with IRES and fluorescent markers enable the selection of transduced LCLs. Based on the HIV-SFFV-mRFP-WPRE vector, the present study constructed vectors to allow expression of one transgene of interest, and in addition, a fluorescent marker protein for identification and selection of transduced cells. In order to enable the coordinated and efficient expression of a transgene and fluorescent marker directly from the SFFV promoter, attenuated IRES was introduced in front of the fluorescent marker. Since a gene located behind the IRES is expected to be translated with lower efficiency than the one in front, such conformation ensures high-level expression of the GOI, rather than the fluorescent marker gene. As transgenes, the present study introduced genes encoding XBP-1 s and BiP proteins (Fig. 6). Flow cytometric analysis and sorting of Raji cells (Fig. 7A) or LCLs (Fig. 7B) was conducted 10 days post-transduction. Notably, the levels of transgene expression with GFP-encoding vectors was higher in Raji cells compared with in LCLs, whereas the levels of transgene expression with RFP-encoding vectors were comparable between the cell lines (Fig. 7). Finally, after sorting, it was demonstrated that LCLs were successfully modified to overexpress the XBP-1 s protein, as confirmed with western blotting (Fig. 8).

\section{Discussion}

The present study aimed to identify a eukaryotic promoter allowing for efficient and stable transduction of normal human B cells with GOI. Quiescent cells are notoriously resistant to various methods of gene transfer. Normal human B cells are particularly difficult to transduce. Nonviral transfection approaches are completely ineffective, and the use of viral vectors requires complex genetic engineering to generate 
pseudotyped lentiviruses. The present study aimed to evaluate the levels of transgene expression with lentiviral vectors containing various promoters, including CMV,EF1 $\alpha$ and SFFV. However, despite satisfactory (CMV and $\mathrm{EF} 1 \alpha)$ and notable (SFFV) levels of transgene expression (Fig. 4), allowing for modification of $\sim 90 \%$ of Raji cells, $<10 \%$ of normal human B cells were transduced with vectors containing these promoters (Fig 2B). Since Raji cells are immortalized B cell-derived lymphoma cells carrying the latent EBV genome, the present study aimed to investigate whether EBV infection of normal $B$ cells will make them more sensitive to lentiviral transduction. Notably, infection of B cells with EBV resulted in the generation of LCLs that could be effectively transduced with lentiviruses carrying the SFFV promoter (Fig. 5). Therefore, these results confirmed the findings of a previous study, which indicated that B cells stimulated with EBV are easily modified with lentiviruses, whereas B cells activated with CD40L and cytokines are resistant to transduction (17). To produce lentiviral particles the 2nd generation packaging vector psPAX2 and the VSVG-expressing envelope vector pMD2.G were used. Using VSVG-lentiviruses the present study managed to modify lymphoma cells and LCLs with high efficacy; however, the present study failed to transduce quiescent normal B cells. It has previously been reported that lentiviruses pseudotyped with the Edmonston MV hemagglutinin and fusion glycoproteins (Hgps and Fgps) allowed for efficient transduction of quiescent human $\mathrm{B}$ and $\mathrm{T}$ cells (12). These findings strongly suggested that the transduction efficiency may be determined at the level of vector entry. It may be hypothesized that bicistronic vectors, when used with lentiviral vectors pseudotyped with the Edmonston MV Hgps and Fgps can serve as a potent transduction tool for normal B cells.

As proof-of-concept, the present study used bicistronic vectors containing genes encoding BiP or XBP-1, as well as fluorescent reporter proteins $\mathrm{mRFP}$ or GFP, and demonstrated that SFFV promoter-based lentiviruses may be used for stable transduction of LCLs with GOI. In conclusion, the present study demonstrated that it is possible to achieve an efficient and stable lentiviral transduction of LCLs using SFFV promoter constructs. This approach may be used in future studies aimed at studying various processes associated with B-cell physiology. Such bicistronic vectors expressing fluorescent proteins (GFP or mRFP) also allow for tracking of modified cells in $e x$ vivo or in vivo studies.

\section{Acknowledgements}

The present study was supported by the National Centre for Research and Development (grant no. INNOTECH-K1/IN1/51/159542/NCBR/12).

\section{References}

1. Kay MA, Glorioso JC and Naldini L: Viral vectors for gene therapy: The art of turning infectious agents into vehicles of therapeutics. Nat Med 7: 33-40, 2001.
2. Naldini L, Blömer U, Gallay P, Ory D, Mulligan R, Gage FH, Verma IM and Trono D: In vivo gene delivery and stable transduction of nondividing cells by a lentiviral vector. Science 272 : 263-267, 1996

3. Jaleco AC, Stegmann AP, Heemskerk MH, Couwenberg F, Bakker AQ, Weijer K and Spits H: Genetic modification of human B-cell development: B-cell development is inhibited by the dominant negative helix loop helix factor Id3. Blood 94: 2637-2646, 1999.

4. Serafini M, Naldini L and Introna M: Molecular evidence of inefficient transduction of proliferating human B lymphocytes by VSV-pseudotyped HIV-1-derived lentivectors. Virology 325: 413-424, 2004.

5. Kvell K, Nguyen TH, Salmon P, Glauser F, Werner-Favre C, Barnet M, Schneider P, Trono D and Zubler RH: Transduction of CpG DNA-stimulated primary human B cells with bicistronic lentivectors. Mol Ther 12: 892-899, 2005.

6. Janssens W, Chuah MK, Naldini L, Follenzi A, Collen D, Saint-Remy JM and VandenDriessche T: Efficiency of onco-retroviral and lentiviral gene transfer into primary mouse and human B-lymphocytes is pseudotype dependent. Hum Gene Ther 14: 263-276, 2003.

7. Yang L, Bailey L, Baltimore D and Wang P: Targeting lentiviral vectors to specific cell types in vivo. Proc Natl Acad Sci USA 103: 11479-11484, 2006

8. Burns JC, Friedmann T, Driever W, Burrascano M and Yee JK: Vesicular stomatitis virus $\mathrm{G}$ glycoprotein pseudotyped retroviral vectors: Concentration to very high titer and efficient gene transfer into mammalian and nonmammalian cells. Proc Natl Acad Sci USA 90: 8033-8037, 1993.

9. Finkelshtein D, Werman A, Novick D, Barak S and Rubinstein M: LDL receptor and its family members serve as the cellular receptors for vesicular stomatitis virus. Proc Natl Acad Sci USA 110: 7306-7311, 2013

10. Amirache F, Lévy C, Costa C, Mangeot PE, Torbett BE, Wang CX, Nègre D, Cosset FL and Verhoeyen E: Mystery solved: VSV-G-LVs do not allow efficient gene transfer into unstimulated T cells, B cells, and HSCs because they lack the LDL receptor. Blood 123: 1422-1424, 2014.

11. Frecha C, Costa C, Lévy C, Nègre D, Russell SJ, Maisner A Salles G, Peng KW, Cosset FL and Verhoeyen E: Efficient and stable transduction of resting B lymphocytes and primary chronic lymphocyte leukemia cells using measles virus gp displaying lentiviral vectors. Blood 114: 3173-3180, 2009.

12. Frecha C, Lévy C, Costa C, Nègre D, Amirache F, Buckland R, Russell SJ, Cosset FL and Verhoeyen E: Measles virus glycoprotein-pseudotyped lentiviral vector-mediated gene transfer into quiescent lymphocytes requires binding to both SLAM and CD46 entry receptors. J Virol 85: 5975-5985, 2011.

13. Funke S, Maisner A, Mühlebach MD, Koehl U, Grez M, Cattaneo R, Cichutek K and Buchholz CJ: Targeted cell entry of lentiviral vectors. Mol Ther 16: 1427-1436, 2008.

14. Dörig RE, Marcil A, Chopra A and Richardson CD: The human CD46 molecule is a receptor for measles virus (Edmonston strain). Cell 75: 295-305, 1993.

15. Tatsuo H, Ono N, Tanaka K and Yanagi Y: SLAM (CDw150) is a cellular receptor for measles virus. Nature 406: 893-897, 2000.

16. Sambrook J, Fritsch EF and Maniatis T: Molecular Cloning: A laboratory manual, 2nd (ed)., Cold Spring Harbor Laboratory Press, Cold Spring Harbor, NY, pp1.63-1.70, 1989.

17. Bovia F, Salmon P, Matthes T, Kvell K, Nguyen TH, Werner-Favre C, Barnet M, Nagy M, Leuba F, Arrighi JF, et al: Efficient transduction of primary human B lymphocytes and nondividing myeloma $\mathrm{B}$ cells with HIV-1-derived lentiviral vectors. Blood 101: 1727-1733, 2003.

18. Heath E, Begue-Pastor N, Chaganti S, Croom-Carter D, Shannon-Lowe C, Kube D, Feederle R, Delecluse HJ, Rickinson $\mathrm{AB}$ and Bell AI: Epstein-Barr virus infection of naïve $B$ cells in vitro frequently selects clones with mutated immunoglobulin genotypes: Implications for virus biology. PLoS Pathog 8: e1002697, 2012.

19. Joseph AM, Babcock GJ and Thorley-Lawson DA: EBV persistence involves strict selection of latently infected B cells. J Immunol 165: 2975-2981, 2000. 